\title{
Inverse geometry for grating-based $x$-ray phase-contrast imaging
}

Tilman Donath, ${ }^{1, a)}$ Michael Chabior, ${ }^{2}$ Franz Pfeiffer, ${ }^{3}$ Oliver Bunk, ${ }^{1}$ Elena Reznikova, ${ }^{4}$ Juergen Mohr, ${ }^{4}$ Eckhard Hempel, ${ }^{5}$ Stefan Popescu, ${ }^{5}$ Martin Hoheisel, ${ }^{5}$ Manfred Schuster, ${ }^{2}$ Joachim Baumann, ${ }^{2}$ and Christian David ${ }^{1}$

${ }^{1}$ Paul Scherrer Institut, 5232 Villigen PSI, Switzerland

${ }^{2}$ Corporate Technology, Siemens AG, 80200 München, Germany

${ }^{3}$ Department of Physik (E17), Technische Universität München, 85748 Garching, Germany

${ }^{4}$ Institute of Microstructure Technology, Forschungszentrum Karlsruhe, Postfach 3640,

76021 Karlsruhe, Germany

${ }^{5}$ Healthcare Sector, Siemens AG, 91301 Forchheim, Germany

(Received 19 June 2009; accepted 22 July 2009; published online 11 September 2009)

Phase-contrast imaging using conventional polychromatic $\mathrm{x}$-ray sources and grating interferometers has been developed and demonstrated for x-ray energies up to $60 \mathrm{keV}$. Here, we conduct an analysis of possible grating configurations for this technique and present further geometrical arrangements not considered so far. An inverse interferometer geometry is investigated that offers significant advantages for grating fabrication and for the application of the method in computed tomography (CT) scanners. We derive and measure the interferometer's angular sensitivity for both the inverse and the conventional configuration as a function of the sample position. Thereby, we show that both arrangements are equally sensitive and that the highest sensitivity is obtained, when the investigated object is close to the interferometer's phase grating. We also discuss the question whether the sample should be placed in front of or behind the phase grating. For CT applications, we propose an inverse geometry with the sample position behind the phase grating. (C) 2009 American Institute of Physics. [doi:10.1063/1.3208052]

\section{INTRODUCTION}

In conventional x-ray imaging, contrast is achieved by the attenuation in an absorbing object. Over the past decade, several techniques have been developed to exploit the contrast given by the phase shift in the object and to overcome the experimental difficulties associated with measuring this effect. Unlike other phase-contrast techniques, ${ }^{1-6}$ the recently developed grating-based differential phase-contrast (DPC) technique works with both highly brilliant but also with conventional, polychromatic laboratory $\mathrm{x}$-ray sources. ${ }^{7-13}$ The latter makes it a suitable candidate for the use in medical and industrial computed tomography (CT) scanners. One of the most stringent difficulties in DPC imaging is the fabrication of suitable gratings with small structures and high aspect ratios. ${ }^{14,15}$ In this paper, an alternative grating setup is presented that relaxes the requirements on the gratings needed and thus facilitates the use of the technique in large-scale scanners.

The interferometer's angular sensitivity changes as a function of the object position within the grating interferometer. To find the optimal object position for the various interferometer setups, a general formula describing the angular sensitivity is derived and validated by measurements using a simple phase phantom. Based on this formula, we discuss the optimal object position with special emphasis on potential phase-contrast CT scanners.

\footnotetext{
${ }^{a)}$ Electronic mail: tilman.donath@psi.ch.
}

\section{MATERIALS AND METHODS}

\section{A. Differential phase signal}

Following Ref. 7, the phase shift $\Phi(x, y)$ imposed on the wave field by an object positioned in the beam path causes a refraction of the beam in $x$-direction by an angle $\alpha$, which is connected with the object's differential phase shift $\partial \Phi / \partial x$ by

$$
\alpha=\frac{\lambda}{2 \pi} \frac{\partial \Phi}{\partial x}
$$

with the $\mathrm{x}$-ray wavelength $\lambda$ and the beam propagating along the $z$-direction. In order to measure the differential phase shift in form of the beam refraction angle $\alpha$, we utilize a Talbot grating interferometer comprising of an absorbing source grating (G0), a phase grating (G1), and an absorbing analyzer grating $(\mathrm{G} 2)$. The central phase grating $\mathrm{G} 1$ of period $p_{1}$ is designed to introduce a phase shift on the incoming wave field at the grating lamellas, which creates an interference pattern of period $p_{2}$ behind G1. The principle of DPC imaging is to measure the beam refraction caused by the object and thus the object's differential phase shift by measuring the displacement of the interference fringes. As the interference fringes cannot be spatially resolved with a conventional $\mathrm{x}$-ray detector, the measurement is carried out using a phase-stepping technique. ${ }^{7,16}$ The intensity oscillation behind $\mathrm{G} 2$ during a lateral stepping scan of one of the gratings is recorded and the fringe displacement is determined in terms of the phase shift $\Delta \varphi(x, y)$ of the oscillation curve for each detector pixel.

The measured phase shift $\Delta \varphi(x, y)$ of the intensity oscillation in each detector pixel is related to the local refraction 
angle $\alpha(x, y)$, the distance $d$ between the two gratings $\mathrm{G} 1$ and $\mathrm{G} 2$, and the period $p_{2}$ of the analyzer grating $\mathrm{G} 2 \mathrm{by}^{7}$

$$
\Delta \varphi=2 \pi \alpha \frac{d}{p_{2}} .
$$

This formula is valid for a flat object positioned in the plane of grating G1. It is also valid in the parallel-beam geometry, if the object is placed upstream of G1. In Sec. II D, we will derive a more general formula.

\section{B. Grating interferometer formulas}

For monochromatic plane waves, i.e., in the parallelbeam case, maximum or minimum modulation of the interference fringes behind G1 is achieved at the fractional Talbot distances,

$$
D_{n}=n \frac{p_{1, e}^{2}}{2 \lambda},
$$

where $n=0,1,2, \ldots$ is an integer number called the Talbot order and $p_{1, e}$ is the effective period of grating G1, i.e., the period of intensity modulation behind G1, which we define as

$$
p_{1, e}=p_{1} / \eta
$$

with $\eta=1$ for a $\pi / 2$-shifting phase grating or an absorption grating and $\eta=2$ for a $\pi$-shifting phase grating. The maximum contrast of the intensity modulation is achieved for an absorption grating at even Talbot orders $(n=0,2,4, \ldots)$ and for a phase grating at odd Talbot orders $(n=1,3,5, \ldots)$.

For spherical waves, i.e., in a cone-beam geometry, the Talbot distances rescale according to the Fresnel scaling theorem $^{17}$ as

$$
d_{n}=M D_{n},
$$

with the magnification factor $M$ of the interference pattern at the position of $\mathrm{G} 2$ :

$$
M=\frac{l+d}{l},
$$

with the source-point-to-G1 distance $l$ and the G1-to-G2 distance $d . M$ is the geometrical magnification for the projection of G1 onto G2 as seen from a source point in G0. Note that generally $M$ is not the same as the geometrical magnification of the object, which is defined by the position of source, object, and detector. Substituting Eq. (6) into Eq. (5) for $d$ $=d_{n}$ and separating the variables, we obtain

$$
\frac{1}{D_{n}}=\frac{1}{l}+\frac{1}{d_{n}},
$$

which is similar to the classical lens equation in optical imaging. Solving for the rescaled Talbot distance $d_{n}$, we find

$$
d_{n}=\frac{l D_{n}}{l-D_{n}} .
$$

The period of G2 is chosen equal to the period of the interference pattern, which is given by

$$
p_{2}=M p_{1, e},
$$

with the geometrical magnification $M$ from Eq. (6). In the parallel-beam case $(l \rightarrow \infty)$, the magnification factor becomes $M=1$ and we obtain $p_{2}=p_{1, e}$.

A third absorbing source grating $\mathrm{G} 0$ of period $p_{0}$ allows to use the interferometer with conventional $\mathrm{x}$-ray sources ${ }^{8,9}$ that, without this additional grating, would not provide sufficient lateral coherence because of their large source size. In the measurements presented here, an x-ray source with a focal spot of $1.0 \mathrm{~mm}$ diameter was used. In the first approximation, the source grating is considered as an array of mutually incoherent line sources. To observe well-defined interference patterns in the G2 plane, the interference images resulting from each line source of the G0 grating must overlap in space, i.e., they must be in registry. ${ }^{18}$ In this case, the interference patterns are constructively superposed, blurred only by the slit profile of a single slit (see Ref. 9). For the G0-to-G1 distance $l$, the G1-to-G2 distance $d$, and $s=l+d$ the total length of the interferometer, the interference patterns from the line sources of G0 are constructively superposed in the plane of $\mathrm{G} 2$, if the periods of gratings $\mathrm{G} 0$ and $\mathrm{G} 2$ are related by ${ }^{8}$

$$
p_{0}=\frac{l}{d} p_{2}
$$

The grating interferometer can either be used with an absorbing source grating G0 of period $p_{0}$ or it can be used with a microfocus source. The width of the source point or the slits, respectively, should be smaller than $p_{0}$ to maintain coherence. In the presented measurement, the slit width of G0 was $p_{0} / 2$.

\section{Interferometer geometries: Conventional, symmetric, and inverse}

For a given total length $s=l+d_{n}$ of the setup and substituting $d_{n}=s-l$ into Eq. (7), we obtain a quadratic equation for $l$ :

$$
l^{2}-s l+s D_{n}=0
$$

with the solutions

$$
l_{1,2}=\frac{s}{2} \pm \sqrt{\frac{s^{2}}{4}-s D_{n}} .
$$

The discriminant in Eq. (12) allows the distinction of the following three cases: (1) For $s<4 D_{n}$, the discriminant is negative and there is no solution. (2) For $s=4 D_{n}$, we obtain a single solution, which corresponds to a symmetric setup with $l=d_{n}=s / 2=2 D_{n}$. (3) For $s>4 D_{n}$, there are two possible solutions, one with $d_{n}<l$ and one with $l<d_{n}$.

The three possible solutions are shown schematically in Fig. 1. The symmetric geometry [Fig. 1(c)] with $l=d$ gives the shortest possible interferometer that can be realized for a given period $p_{1}$, with a total length of $s=4 D_{n}$. Additionally, it has the advantage that both absorption gratings G0 and G2 have the same period $p_{0}=p_{2}$ and can be produced using the same fabrication process. Recently, we have demonstrated this geometry for a $\pi$-shifting phase grating, for which all 

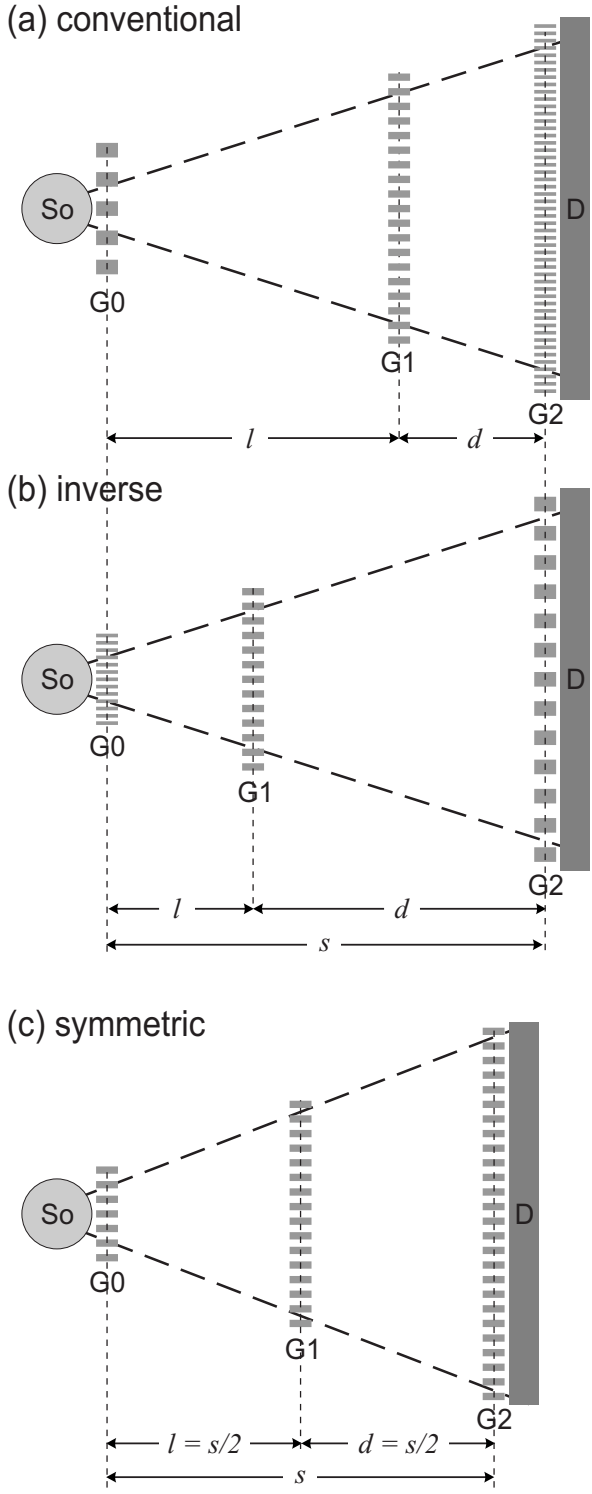

FIG. 1. Grating interferometer geometries for given phase-grating period $p_{1}$. The interferometer consists of source grating (G0), phase grating (G1), and analyzer grating (G2). The total length $s$ is the distance between G0 and G2, which is smaller than the distance between source (So) and detector (D). (a) Conventional geometry with $l>d$ and $p_{0}>p_{1}>p_{2}$, (b) inverse geometry with $l<d$ and $p_{0}<p_{1}<p_{2}$, and (c) symmetric geometry with $l=d$ and $p_{0}$ $=p_{1}=p_{2}$ (for a $\pi$-shifting phase grating G1). For given $p_{1}$, the symmetric geometry has the shortest possible total length.

grating periods including that of $\mathrm{G} 1$ become equal with $p_{0}$ $=p_{1}=p_{2} .{ }^{19}$ From the nonsymmetric solutions, only the case $d<l$ has been considered in previous publications. ${ }^{7,16}$ In the following, this case will be referred to as the conventional geometry [Fig. 1(a)]. For the second solution with $l<d$, the phase grating is closer to the source grating than to the analyzer grating. The distances $l$ and $d$ are interchanged and, consequently, also the grating periods of G0 and G2 are interchanged. Thus, we will refer to the solution with $l<d$ as the inverse geometry [Fig. 1(b)], since the inverse geometry is equivalent to the conventional geometry with reversed beam direction. This tells us that the three-grating interferometer can be used in either direction.

The inverse geometry with $l<d$ offers the following practical advantages over the conventional geometry in the realization of $\mathrm{x}$-ray grating interferometers

- The grating with the finest structures, and thus the grating which is most challenging to produce by microfabrication, is G0. It has a much smaller total size than G2 because of the high geometrical magnification. The grating with the largest area and the coarsest structures is now G2, which presents the least stringent requirements for microfabrication.

- Since the period of G2 increases, the grating lamellas can be easily made higher so that the use of other materials than gold that are less dense and less expensive can be considered. This offers advantages with respect to the transmission curve, process compatibility, and production costs.

- As grating G1 moves closer to the source, its dimensions can be reduced as well.

Apart from the grating arrangement, we have to choose the object position within the grating interferometer. In previous publications, the object was placed between source (or G0) and G1. This was practical, since there is more space in front of the G1 grating in the so far realized conventional geometry with $d<l$. For the inverse geometry, with $l<d$, it is favorable to place the object behind G1 (compare Fig. 1). However, other applications such as laboratory micro-CT may still require the object to be close to the source in order to profit from the geometrical magnification in a cone-beam setup. In the following, a general formula for the interferometer's angular sensitivity as a function of the object position is derived.

\section{Angular sensitivity of the grating interferometer}

We define the angular sensitivity of the interferometer as

$$
S:=\frac{1}{2 \pi} \frac{\Delta \varphi}{\alpha},
$$

describing the ratio of phase-shift $\Delta \varphi$ measured in the intensity oscillation of the phase scan (normalized to $2 \pi$ ) to the refraction angle $\alpha$ from Eq. (1) caused by the object. The sensitivity $S$ clearly not only depends on the interferometer geometry and grating periods but also on the position of the object relative to the gratings. Here, we derive the sensitivity allowing for a variable object position from pure geometrical considerations. For a special case, namely, for a two-grating interferometer using a microfocus source and with the object positioned between G0 and G1, the angular sensitivity has been derived by Engelhardt. ${ }^{16}$ The angular sensitivity linearly decreases from its maximum to zero, when the object is moved from the G1 plane toward the source.

Here, we derive a more general formula for the angular sensitivity that describes the two cases with object behind and in front of the phase grating G1 based on the respective schemes in Figs. 2(a) and 2(b). According to this figure, the object position $r$ is defined relative to the phase grating $\mathrm{G} 1$, thus distinguishing between the case of negative $r$ with the object between source point and G1 $(-l \leq r \leq 0)$ and the case of positive $r$ with the object between G1 and G2 $(0 \leq r$ $\leq d$ ). 


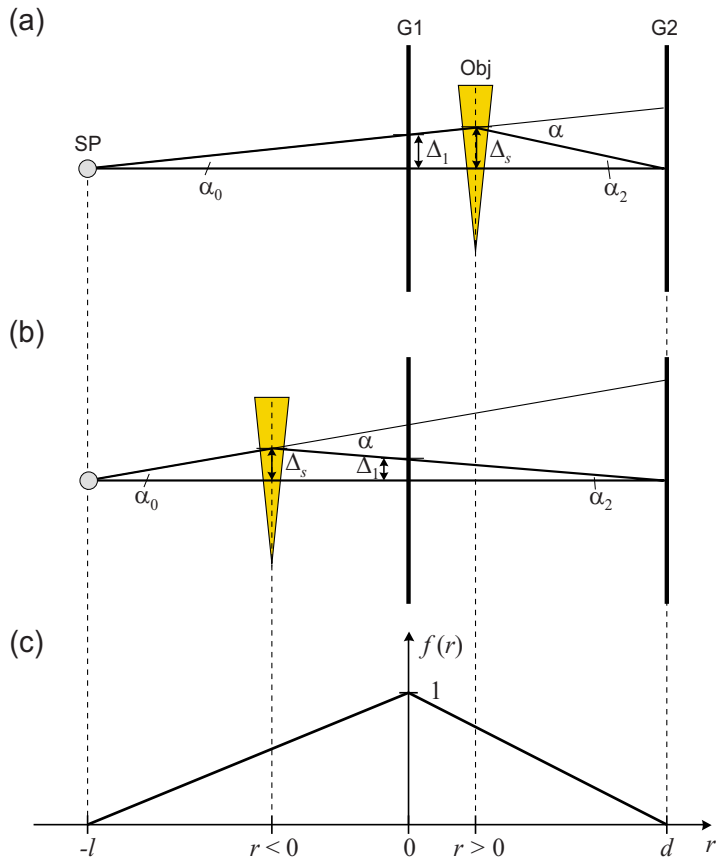

FIG. 2. (Color online) Beam path for calculation of angular sensitivity as a function of object position. The relative sensitivity $f(r)$ as a function of object position $r$ is derived distinguishing the cases: (a) refracting object between $\mathrm{G} 1$ and G2 with $0 \leq r \leq d$ and (b) refracting object between source point and G1 with $-l \leq r \leq 0$. (c) Plot of $f(r)$, which describes the linear scaling of the sensitivity. SP: source point; Obj: object; G1: phase grating; G2: analyzer grating. The source point can either be the focal spot of a microfocus x-ray tube or a slit in a G0 grating.

The rays from a source point, practically realized as either the spot of a microfocus tube or a slit in the source grating G0, project the G1 onto the G2 grating. Let us consider a purely refractive flat object, symbolized by the wedge in Figs. 2(a) and 2(b) that changes the beam propagation direction by a given refraction angle $\alpha$. The wedge displaces the projection of G1 in the G2 plane. Or in other words, a different point of G1 is projected onto the same point of G2, when the wedge is inserted. Thus, a lateral displacement of G1 by $\Delta_{1}$ is needed to recover the same projection, i.e., to let the ray through the wedge project the same point of G1 onto $\mathrm{G} 2$ as for the central undisturbed ray. The displacement $\Delta_{1}$ is directly related to the phase shift $\Delta \varphi$ measured in the DPC phase scans as

$$
\frac{\Delta \varphi}{2 \pi}=\frac{\Delta_{1}}{p_{1, e}},
$$

where $p_{1, e}$ is the effective period of grating G1 defined in Eq. (4).

First, we will determine the displacement $\Delta_{1}$ for the case $0 \leq r \leq d$ shown in Fig. 2(a). From the figure, we see that the displacement is given by $\Delta_{1}=\tan \left(\alpha_{0}\right) l \approx \alpha_{0} l$, where the angle $\alpha_{0}$ is an auxiliary quantity that we have to determine and the tangent function for the minute refraction angles in x-ray imaging has been approximated here with the angle in radians. The angles $\alpha_{0}$ and $\alpha_{2}$ are geometrically related by the two equations:

$$
\alpha=\alpha_{0}+\alpha_{2}
$$

$$
\left(\Delta_{s}=\right) \alpha_{0}(l+r)=\alpha_{2}(d-r),
$$

where the auxiliary quantity $\Delta_{s}$ shown in Fig. 2(a) is the beam separation of the undisturbed and the refracted beam at the object position. Note that we have again approximated the tangent with the angle in radians and that these equations are valid for both cases $(r \leq 0$ and $r \geq 0)$. Solving Eqs. (15) and (16) for $\alpha_{0}$, we obtain the displacement

$$
\Delta_{1}=\alpha_{0} l=\alpha\left(\frac{1}{l}+\frac{1}{d}\right)^{-1}\left(1-\frac{r}{d}\right) \text { for } 0 \leq r \leq d .
$$

For the second case with $-l \leq r \leq 0$ shown in Fig. 2(b), we determine the displacement $\Delta_{1}=\tan \left(\alpha_{2}\right) d \approx \alpha_{2} d$. Since the displacement now depends on $\alpha_{2}$, we solve Eqs. (15) and (16) for $\alpha_{2}$ and obtain

$$
\Delta_{1}=\alpha_{2} d=\alpha\left(\frac{1}{l}+\frac{1}{d}\right)^{-1}\left(1+\frac{r}{l}\right) \text { for } \quad-l \leq r \leq 0 .
$$

Combining Eqs. (17) and (18), we find for the angular sensitivity

$$
S=\frac{1}{2 \pi} \frac{\Delta \varphi}{\alpha}=\left(\frac{1}{l}+\frac{1}{d}\right)^{-1} \frac{1}{p_{1, e}} f(r),
$$

with the function

$$
f(r)=\left\{\begin{array}{rll}
1+\frac{r}{l} & \text { for } & -l \leq r \leq 0 \\
1-\frac{r}{d} & \text { for } & 0 \leq r \leq d .
\end{array}\right.
$$

Thus, we have separated the sensitivity into an interferometer-specific factor and the triangular, linear scaling function $f(r)$, plotted in Fig. 2(c), that only depends on the relative object position within any interferometer. The function $f(r)$ reaches its maximum of unity for $r=0$, i.e., for the object in the plane of G1 and falls off linearly, when the object is moved toward G0 or G2. For $r=-l$ and $r=d$, i.e., when the object is in the plane of G0 or G2, respectively, we have $f(r)=0$ and, thus, zero sensitivity.

Hence the interferometer-specific factor gives the interferometer's maximum sensitivity

$$
S_{\max }:=S(r=0)=\left(\frac{1}{l}+\frac{1}{d}\right)^{-1} \frac{1}{p_{1, e}}=\frac{d}{M} \frac{1}{p_{1, e}}=\frac{d}{p_{2}},
$$

where we have simplified the term in parentheses using the definition of the magnification factor $M$ in Eq. (6) and the relation $p_{2}=M p_{1, e}$ in Eq. (9). Furthermore, we obtain from Eq. (5) for $d=d_{n}=M D_{n}$ and Eq. (3) that $S_{\max }=D_{n} / p_{1, e}$ $=n p_{1, e} / 2 \lambda$. Thus, we observe an increase in sensitivity with increasing G1 period $p_{1}$, which comes at the cost of an increased Talbot distance $d \propto p_{1, e}^{2}$ between G1 and G2. Introducing $S_{\max }$, we can rewrite Eq. (19) as

$$
S=S_{\max } f(r) .
$$

Thus, for the case of a flat object in the plane of G1, we obtain the maximum sensitivity $S=S_{\max }=d / p_{2}$, which is consistent with Eq. (2), i.e., with the result of Weitkamp et al. ${ }^{7}$ 
Using the relation $p_{0}=(l / d) p_{2}$ from Eq. (10), one can also see directly that the maximum sensitivity $S_{\max }=d / p_{2}$ $=l / p_{0}$ is identical for a conventional and an inverse setup using the same gratings, i.e., when the parameters $l$ and $d$ as well as $p_{0}$ and $p_{2}$ are interchanged. Thus we can rewrite Eq. (21) in a fully symmetric form as

$$
S_{\max }=\frac{l}{p_{0}}=\left(\frac{1}{l}+\frac{1}{d}\right)^{-1} \frac{1}{p_{1, e}}=\frac{d}{p_{2}} .
$$

So far, we have assumed that the phase grating G1 is used for the phase-stepping scan. However, we do obtain the same result for phase stepping with G0 or G2. This can be easily derived looking at the displacements $\Delta_{0}$ and $\Delta_{2}$ of the wave field in the planes of G0 and G2, respectively, similar to the above derivation. This results in the more general form of Eq. (14):

$$
\frac{\Delta \varphi}{2 \pi}=\frac{\Delta_{0}}{p_{0}}=\frac{\Delta_{1}}{p_{1, e}}=\frac{\Delta_{2}}{p_{2}},
$$

where $\Delta \varphi$ is the phase shift measured using either grating.

\section{E. Measurement of the angular sensitivity}

To compare the sensitivity of the conventional and the inverse grating geometry experimentally and to confirm the formula derived for the angular sensitivity in Eq. (22), the phase shift $\Delta \varphi$ was measured for both geometries as a function of object position $r$ using a simple phase object. For both geometries, nine object positions in front of the phase grating and another nine object positions behind the phase grating were recorded.

The experiments were carried out at a laboratory setup at the Paul Scherrer Institut (PSI) in Villigen, Switzerland. As an X-ray source, a COMET MXR-160HP/11 tube with a tungsten anode and a target angle of $11^{\circ}$ was used. It was operated at an anode voltage of $65 \mathrm{kV}$, an anode current of $10 \mathrm{~mA}$, and with an effective focus diameter of $1 \mathrm{~mm}$. Low energies in the spectrum were removed using a $10 \mathrm{~mm}$ thick aluminum filter. The detector consisted of three main components: (1) a Hamamatsu cesium iodide (CsI) scintillator of $600 \mu \mathrm{m}$ thickness, (2) an optical lens and mirror system, and (3) a charge-coupled device camera (model Proline of Finger Lakes Instrumentation) with $1024 \times 1024$ pixels of $24 \times 24 \mu \mathrm{m}^{2}$, which was read in $2 \times 2$ binning mode. The phase grating G1 was manufactured at the Laboratory for Micro- and Nanotechnology of PSI in silicon (Si) with lamellas of $61 \mu \mathrm{m}$ height and a period of $6.3 \mu \mathrm{m}$. The absorbing gold $\mathrm{Au}$ ) gratings were manufactured at the PSI and at the Forschungszentrum Karlsruhe with periods of 8.5 and $5.0 \mu \mathrm{m}$ and structure heights of 90 and $120 \mu \mathrm{m}$, respectively. ${ }^{14,15} \mathrm{G} 1$ produces a relative phase shift of $\pi$ at the design energy of $47.6 \mathrm{keV}$. According to the interferometer formulas in Secs. II B and II C, this design energy results in intergrating distances of $l=514 \mathrm{~mm}$ and $d$ $=303 \mathrm{~mm}$ for the conventional setup and, vice versa, in distances of $l=303 \mathrm{~mm}$ and $d=514 \mathrm{~mm}$ for the inverse setup.

For each projection image, a phase-stepping scan without the phase object in the beam (reference scan) and a sample scan with the object in the beam were recorded.
Phase stepping was carried out with the G1 grating in eight steps over one period of G1 (two effective periods $p_{1, e}$ ) and each image was recorded with $4.5 \mathrm{~s}$ exposure time. The images were finally cropped to a size of $120 \times 295$ (horiz. $\times$ vert.) pixels.

As a well-defined phase object, a homogeneous aluminum rod with $5 \times 5 \mathrm{~mm}^{2}$ square-shaped cross section was used. Its diagonal was aligned along the beam direction, so that in good approximation, the rod exhibits a phase shift like a symmetric triangle or double wedge, with two areas of constant phase gradients of opposite sign. The expected absolute angle of refraction caused by the aluminum rod can be estimated as

$$
|\alpha|=\frac{\lambda}{2 \pi} \frac{\Delta \Phi}{\Delta x}=2 \delta,
$$

where $\delta$ is the refractive index decrement. We have used the fact that the phase shift caused by the rod changes as $\Delta \Phi$ $=(2 \pi / \lambda) \delta 2 \Delta x$, when moving toward the rod center by $\Delta x$. For our design energy of $47.6 \mathrm{keV}$, we determined $\delta$ $=0.238 \times 10^{-6}$ and $|\alpha|=2 \delta=0.477 \mu \mathrm{rad}$ for the aluminum rod. Here, we calculated $\delta$ from the tabulated values of $f_{1}$ for elastic photon-atom scattering, anomalous scattering factors from Ref. 20.

\section{RESULTS AND DISCUSSION}

Images of the phase object recorded at several selected object positions in the inverse geometry are displayed in Fig. 3 . Note that the magnification of the rod decreases with increasing source-to-object distance. The images show the local refraction angles $\alpha(x, y)$, which of course exhibit opposite direction and sign for the left and right half of the rod. For each image, two regions of interest each containing 16 $\times 260$ pixels for the left and the right half of the rod were manually selected, as exemplarily shown in Fig. 3(d), and the phase shift for each side and object position was calculated as the mean over the defined region. The signals from the left and right sides were subtracted and divided by 2 , in order to remove any signal offset, thus obtaining a quantitatively exact phase shift $\Delta \varphi$. Also, the standard deviation of the mean value was determined for each side and, subsequently, the standard variation $\sigma(\Delta \varphi)$ in the phase shift was determined by error propagation. The measured phase shifts are plotted against the object position $r$ in Fig. 4 for both the conventional (a) and the inverse geometry (b). The plots exhibit the expected linear dependence of the measured phase shift on the object position, as described by Eq. (20). From this plot, the phase shift $\Delta \varphi(r=0)$ of the object at the position of the phase grating G1, i.e., for $r=0$, and maximum sensitivity, were extrapolated using linear regression. The obtained values of $\Delta \varphi_{c}=0.162 \pm 0.008 \mathrm{rad}$ for the conventional and $\Delta \varphi_{i}=0.164 \pm 0.003 \mathrm{rad}$ for the inverse setup are equal within the limits of accuracy. The linear regression returns correlation coefficients of 0.990 for the conventional and 0.998 for the inverse setup, clearly showing that the linear change in sensitivity describes the data correctly.

Our measurement was limited mainly by the accuracy of object positioning, while the influence of noise from the im- 
(a) $-250 \mathrm{~mm}$

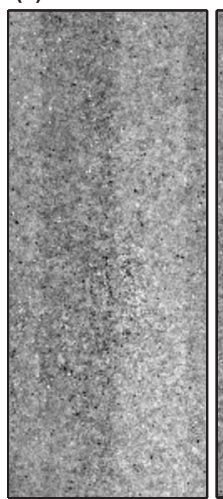

(b) $-120 \mathrm{~mm}$

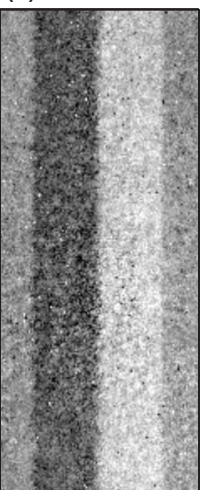

(c) $-10 \mathrm{~mm}$

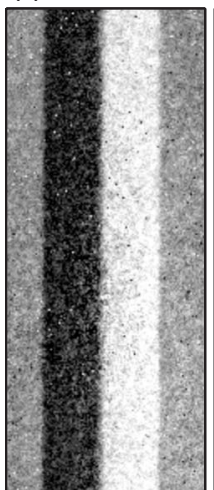

(d) $+78 \mathrm{~mm}$

(e) $+344 \mathrm{~mm}$

(f) $+504 \mathrm{~mm}$

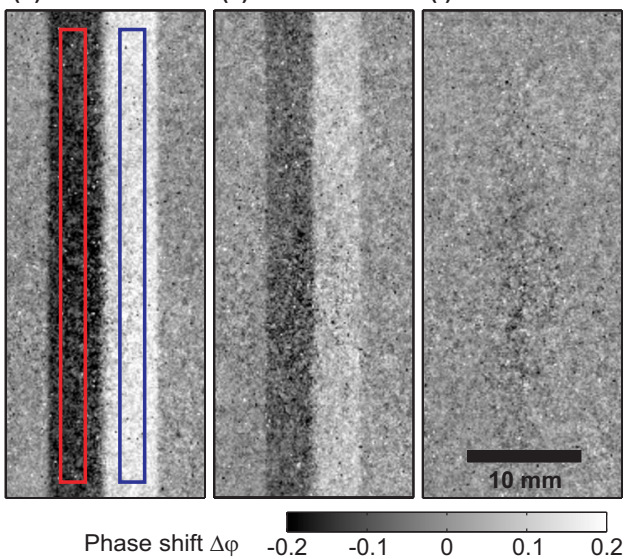

FIG. 3. (Color online) Images of the measured phase shift $\Delta \varphi$ (gray coded) of a square-shaped $5 \times 5 \mathrm{~mm}^{2}$ aluminum rod. The viewing direction is along one of the diagonals of the square-shaped rod. Images measured in the inverse geometry are shown for a few selected object positions $r$ : (a) -250 mm, (b) -120 $\mathrm{mm},(\mathrm{c})-10 \mathrm{~mm},(\mathrm{~d})+78 \mathrm{~mm},(\mathrm{e})+344 \mathrm{~mm}$, and (f) $+504 \mathrm{~mm}$. The gratings G0, G1, and G2 were positioned at $r_{0}=-303 \mathrm{~mm}, r_{1}=0 \mathrm{~mm}$, and $r_{2}=$ $+514 \mathrm{~mm}$, respectively. The extension of the horizontal diagonal is $\sqrt{2} \cdot 5 \mathrm{~mm}=7.07 \mathrm{~mm}$. From (a) to (f), the source-to-rod distance increases, while the geometric magnification of the rod and thus the size of the projection image decrease. The averaging boxes for the left side (red) and the right side (blue) of the rod are exemplarily shown in (d). The images were cropped to a size of $120 \times 295$ (horiz. $\times$ vert.) pixels and the color bar gives the measured phase-shift in radians.

ages was negligible. The object position with the largest error of the determined mean phase shift exhibited a standard deviation of only $\sigma(\Delta \varphi)=0.853 \times 10^{-3} \mathrm{rad}$, which is too small to be plotted, as an error bar in Fig. 4. This standard deviation was determined for the mean value over the regions of interest. The corresponding standard deviation for an individual pixel was determined to be $0.061 \mathrm{rad}$ on the average. This noise level is clearly visible in the images of Fig. 3.
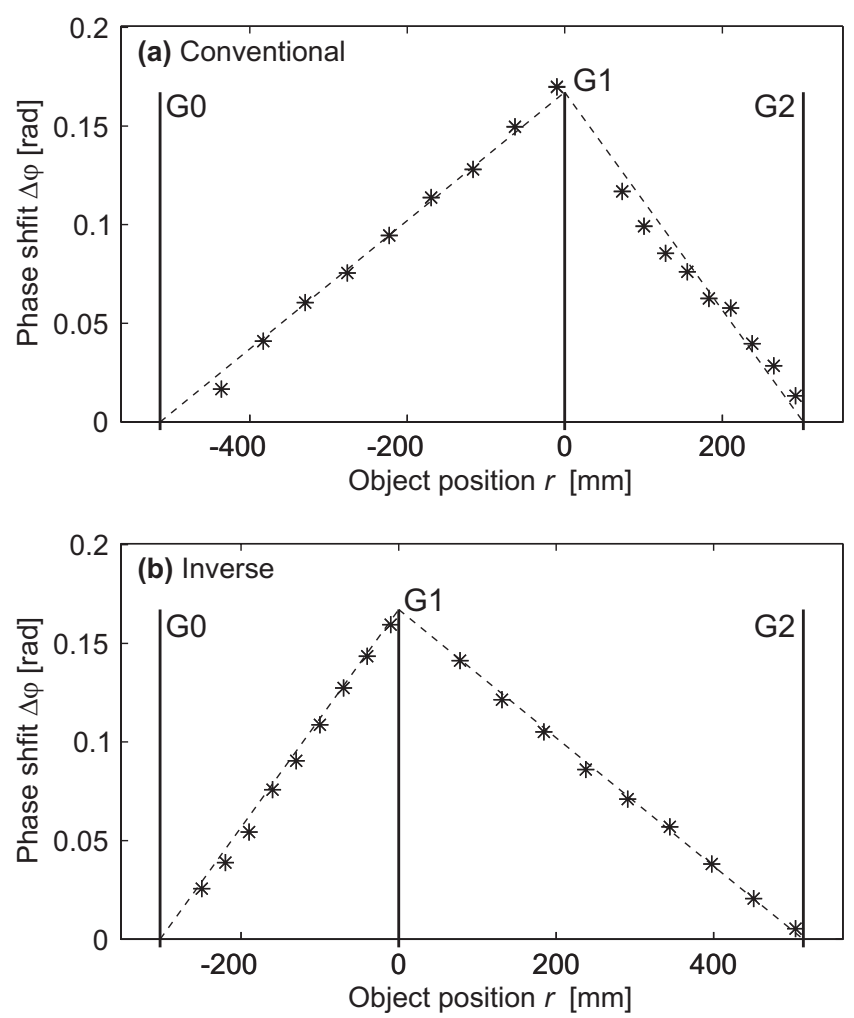

FIG. 4. Phase shift $\Delta \varphi$ measured on a square-shaped $5 \times 5 \mathrm{~mm}^{2}$ aluminum rod at 18 object positions. (a) Conventional geometry with G0 and G2 grating at $r_{0}=-514 \mathrm{~mm}$ and $r_{2}=303 \mathrm{~mm}$ and (b) inverse geometry with G0 and $\mathrm{G} 2$ grating located at $r_{0}=-303 \mathrm{~mm}$ and $r_{2}=514 \mathrm{~mm}$.
One can also compare the measured absolute phase shift with the theoretical prediction for the refraction angle. The extrapolated phase shifts can be converted into refraction angles $\alpha_{i}$ and $\alpha_{c}$ using the definition of $S$ in Eq. (19) according to $\alpha=\left(1 / S_{\max }\right)(\Delta \varphi / 2 \pi)$. For our conventional setup, with $d=303 \mathrm{~mm}$ and $p_{2}=5 \mu \mathrm{m}$, there is $S_{\max }=d / p_{2}$ $\approx 60600$. Thus, we obtain refraction angles of $\alpha_{c}$ $=0.425 \pm 0.021 \mu \mathrm{rad}$ and $\alpha_{i}=0.431 \pm 0.008 \mu \mathrm{rad}$, which are about $10 \%$ smaller than the theoretically expected value of $|\alpha|=0.477 \mu \mathrm{rad}$ calculated at the end of Sec. II E. This difference can be explained by an x-ray spectrum that contributes to the phase measurement, which has an effective energy that is higher than the $47.6 \mathrm{keV}$ assumed in the calculation of the theoretical value. The correct theoretical value of $|\alpha|=0.428 \mu \mathrm{rad}$ (average of $\alpha_{c}$ and $\alpha_{i}$ ) is obtained for an effective $\mathrm{x}$-ray energy of $50.2 \mathrm{keV}$, which is reasonable.

\section{CONCLUSIONS}

Our results clearly show that the conventional and the inverse geometry perform equally well at phase measurements. In many cases, the inverse geometry will be favorable due to its relaxed requirements on grating fabrication.

The dependence of the interferometer sensitivity on the object position is problematic for CT measurements, in which the recorded projections should ideally be line integrals of a constant function that represents an object property. This function must be independent of the projection direction, but in the case of a nonparallel beam, the sensitivity of the interferometer will vary along the x-ray path, such that the signal from a point outside the center of rotation will contribute differently depending on the projection angle. From Eq. (20), we see that it will generally be the better choice to place the object on the side of the phase grating that offers more space, i.e., in front of the phase grating for the conventional geometry and behind the phase grating for the inverse geometry, since this will minimize the relative 
sensitivity change. Generally, for a CT scanner there must be enough space for a sufficiently sized gantry opening. Note that the discriminant in Eq. (12) is also the greatest possible radius for a gantry opening, for which the G1 grating rotates at the position of this gantry opening.

With a total length $s=l+d$ of only $817 \mathrm{~mm}$, the interferometer realized here is remarkably compact, especially for the relatively high design energy of $47.6 \mathrm{keV}$ and a threegrating setup using a sourcing grating in combination with a conventional $\mathrm{x}$-ray tube. To the best of our knowledge, this is the shortest $\mathrm{x}$-ray Talbot interferometer built so far at an $\mathrm{x}$-ray tube.

In summary, we have presented and experimentally demonstrated an inverse geometry for DPC imaging. The dependence of the angular sensitivity on the object position within the interferometer was calculated and measured for both the conventional and the inverse geometry. As expected, the angular sensitivity for both geometries is highest, when the object is in the plane of the phase grating G1, and decays linearly when the object is moved toward the gratings G0 and G2, respectively. The results show that the inverse geometry offers equal sensitivity, while it reduces the requirements on the interferometer. The inverse setup should thus be favorable for the use in medical and industrial CT scanners.

\section{ACKNOWLEDGMENTS}

F.P. acknowledges support through the DFG Cluster of Excellence "Munich-Centre for Advanced Photonics".
${ }^{1}$ U. Bonse and M. Hart, Appl. Phys. Lett. 6, 155 (1965).

${ }^{2}$ L.D. Chapman, W.C. Thomlinson, R.E. Johnston, D. Washburn, E. Pisano, N. Gmuer, Z. Zhong, R. Menk, F. Arfelli, and D. Sayers, Phys. Med. Biol. 42, 2015 (1997).

${ }^{3}$ K.A. Nugent, T.E. Gureyev, D.F. Cookson, D. Paganin, and Z. Barnea, Phys. Rev. Lett. 77, 2961 (1996).

${ }^{4}$ J. F. Clauser, U.S. Patent No. 5,812,629 (September 22, 1998).

${ }^{5}$ A. Momose, Opt. Express 11, 2303 (2003).

${ }^{6}$ A. Momose, Jpn. J. Appl. Phys., Part 1 44, 6355 (2005).

${ }^{7}$ T. Weitkamp, A. Diaz, C. David, F. Pfeiffer, M. Stampanoni, P. Cloetens, and E. Ziegler, Opt. Express 13, 6296 (2005).

${ }^{8}$ F. Pfeiffer, T. Weitkamp, O. Bunk, and C. David, Nat. Phys. 2, 258 (2006).

${ }^{9}$ T. Weitkamp, C. David, C. Kottler, O. Bunk, and F. Pfeiffer, Proc. SPIE, 6318, 63180S (2006).

${ }^{10}$ A. Momose, W. Yashiro, Y. Takeda, Y. Suzuki, and T. Hattori, Jpn. J. Appl. Phys., Part 1 45, 5254 (2006).

${ }^{11}$ F. Pfeiffer, O. Bunk, C. David, M. Bech, G. Le Duc, A. Bravin, and P. Cloetens, Phys. Med. Biol. 52, 6923 (2007).

${ }^{12}$ F. Pfeiffer, C. Kottler, O. Bunk, and C. David, Phys. Rev. Lett. 98, 108105 (2007).

${ }^{13}$ F. Pfeiffer, O. Bunk, C. Kottler, and C. David, Nucl. Instrum. Methods Phys. Res. A 580, 925 (2007).

${ }^{14}$ C. David, J. Bruder, T. Rohbeck, C. Grünzweig, C. Kottler, A. Diaz, O. Bunk, and F. Pfeiffer, Microelectron. Eng. 84, 1172 (2007).

${ }^{15}$ E. Reznikova, J. Mohr, M. Boerner, V. Nazmov, and P. Jakobs, Microsyst. Technol., 14, 1683 (2008).

${ }^{16}$ M. Engelhardt, J. Baumann, M. Schuster, C. Kottler, F. Pfeiffer, O. Bunk, and C. David, Appl. Phys. Lett. 90, 224101 (2007).

${ }^{17}$ D. M. Paganin, Coherent X-Ray Optics (Oxford University Press, New York, 2006).

${ }^{18}$ K. Patorski, Progr. Optics, 27, 3 (1989).

${ }^{19}$ T. Donath, F. Pfeiffer, O. Bunk, W. Groot, M. Bednarzik, C. Grünzweig, E. Hempel, S. Popescu, M. Hoheisel, and C. David, Rev. Sci. Instrum., 80, 053701 (2009).

${ }^{20}$ The scattering factors were taken from the file f1f2_asf_Kissel.dat of the DABAX library (http://ftp.esrf.eu/pub/scisoft/xop2.3/DabaxFiles/). 